\title{
Freunde in der Not
}

\section{Die Bedeutung sozialer Netzwerke bei Krisenvorbeugung und Krisenbewältigung}

\author{
Albert Lenz
}

Crisis are part of life and show that people are in problematic situations. Seen from a scientific perspective, the term crisis is by no means precise. Instead, it describes a situation in which a person is manifestly dealing with limits and fundamental presumptions and conditions are questioned - presumptions and conditions that up to now had been taken for granted. The belong to social networks can hinder individual crisis or belp to overcome them quicker than it would be possible otherwise.

La crise fait partie de la vie et montre que les gens traversent des situations difficiles. D'un point de vue scientifique, le terme de crise n'est en rien précis. Au contraire, il décrit une situation dans laquelle un individu est manifestement et profondément confronté à ses limites et dans laquelle ses présomptions et repères fondamentaux sont remis en question présomptions et repères fondamentaux qui jusque là ont été considérés comme acquis. S'insérer dans des réseaux sociaux peut prévenir l'état de crise ou aider à la surmonter plus rapidement qu'il ne serait possible autrement.

Prof. Dr. Albert Lenz lehrt Klinische Psychologie und Sozialpsychologie an der Katholischen Fachhochschule Nordrhein-Westfalen (vgl. nebenstehenden Autorenhinweis).

E-Mail a.lenz@kfhnw.de
Krisen gehören zum Leben und zeigen, dass Menschen in Not sind. Wissenschaftlich betrachtet ist Krise kein präziser Begriff, sondern beschreibt einen akuten $\mathrm{Zu}$ stand, in dem der Mensch mit Grenzen konfrontiert ist und grundlegende Voraussetzungen in Frage gestellt werden, die bislang für das Leben als gültig erlebt wurden. Die Einbindung in soziale Netzwerke können individuelle Krisen verhindern, mildern oder zumindest schneller überwinden lassen.

In der Praxis haben sich einige Unterscheidungen durchgesetzt, die aber keiner eindeutigen Klassifikation von Krisen folgen (Bergold \& Schürmann, 2001). So spricht man von einer psychosozialen Krise in Abgrenzung von einer psychiatrischen Krise oder einem psychiatrischen Notfall, von suizidaler Krise, von traumatischer Krise in der Folge von unerwarteten und traumatischen Belastungen wie beispielsweise durch schwere Krankheit, Tod einer wichtigen Bezugsperson, Trennung der Eltern oder Gewalterfahrung. Veränderungskrisen werden durch erwartbare Lebensveränderungen wie das Verlassen des Elternhauses, Lehre und Studium oder Umzug ausgelöst. Reifungsund Entwicklungskrisen sind verbunden mit den psychischen, sozialen und biologischen Veränderungen in Kindheit und Jugend sowie mit den Entwicklungsaufgaben, die Kinder und Jugendliche immer wieder vor neue Anforderungen stellen.

Das generelle Kennzeichen von Krisen ist der Verlust des seelischen Gleichgewichts, den ein Mensch verspürt, wenn er mit Ereignissen oder Lebensumständen konfrontiert wird, die er im Moment nicht bewältigen kann, weil sie von der Art und dem Ausmaß her die durch frühere Erfahrungen erworbenen Fähigkeiten und erprobten Mittel zur Erreichung wichtiger Lebensziele oder zur Bewältigung seiner Lebenssituation überfordern (Cullberg, 1978). Krisen gehen einher mit starker Erschütterung und heftigen Ge- fühlen wie Hoffnungslosigkeit und Hilflosigkeit, Verzweiflung, Trauer und Angst, Scham und Schuldgefühle, Selbstzweifel und Einsamkeitsgefühle.

In der Folge kommt es häufig zu Änderungen der Alltagsgewohnheiten, sozialem Rückzug, Flucht in Traumwelten und regressivem Verhalten (Sonneck, 2000). Die Intensität der Krise ist abhängig, von der Art des Krisenanlasses, von der subjektiven Einschätzung des belastenden Lebensereignisses, die wesentlich durch die psychische Verfassung der Person beeinflusst ist sowie insbesondere durch das Vorhandensein oder die Mobilisierbarkeit personaler und sozialer Ressourcen. Menschen, die über ein stabiles Selbstwertgefühl und über ein Gefühl der Selbstwirksamkeit verfügen sowie aktive Problemlösungen anstreben und in ein funktionierendes, Sicherheit vermittelndes interpersonales Beziehungssystem eingebettet sind, werden Krisen besser bewältigen und sie vielleicht als Chance begreifen können, als jemand, der grübelt, an sich selbst zweifelt, zu Problem vermeidenden Strategien neigt und darüber hinaus auf sich gestellt ist.

Die personalen und sozialen Ressourcen bilden gemeinsam das Potenzial der Lebensbewältigung, über das eine Person verfügt. Die personalen Ressourcen üben offensichtlich handlungssteuernde und handlungsregulierende Funktionen aus. Sie greifen ein bei der Bewertung von Situationen bezüglich ihrer Optionen und Restriktionen und bei der Entwicklung von Handlungszielen und als »Vielzweckwerkzeuge " auch im relativ stressfreien Alltag einen starken Einfluss auf das Befinden der Person. Soziale Ressourcen sind psychosoziale Schutzfaktoren, die generell die Anpassung des Individuums an seine Umwelt fördern und die Manifestation einer Störung verhindern oder zumindest erschweren können, und darüber hinaus in Krisen und akuten Belastungssituationen als Puffer oder Polster eine entscheidende Rolle spielen (Lenz, 2002). 


\section{Soziale Netzwerke sind ein Begleitschutz in Krisen}

Soziale Ressourcen sind durch das Vorhandensein eines hilfreichen sozialen Netzwerkes gegeben. Unter einem sozialen Netzwerk versteht man das Geflecht sozialer Beziehungen einer Person zu ihren Familienmitgliedern, Verwandten, Freunden, Bekannten, Nachbarn, Arbeitskollegen und anderen Bezugspersonen. Keupp (1987) spricht von den spezifischen Webmustern unserer alltäglichen Beziehungen.

Soziale Integration und vor allem die verschiedenen Formen sozialer Unterstützung gelten als die wichtigsten Potenziale sozialer Netzwerke. Soziale Integration bezieht sich auf das Vorhandensein und das Ausmaß von Sozialkontakten (Schwarzer \& Leppin 1989). Soziale Unterstützung wird dagegen als sozialer Austauschprozess verstanden, in dem die hilfesuchende und die Hilfe bereitstellende Person mit dem Ziel zusammenwirken, um stresserzeugende Anforderungen und deren emotionalen Folgen zu reduzieren und eine aktive, effiziente Bewältigung der erfahrenen Belastungen zu fördern (Diewald 1991).

Das spezifische Potenzial sozialer Netzwerke liegt in der Vermittlung umfassender sozialer Ressourcen (vgl. Röhrle 1994). Soziale Ressourcen wirken nicht nur als Puffer in Krisensituationen, mildern belastende Lebensereignisse und bilden einen Schutzschild gegenüber Herausforderungen, Spannungszuständen und Stressoren, sondern unterstützen nach Thoits (1994) das individuelle Bewältigungshandeln im Sinne von "coping-assistance «. Netzwerkmitglieder stärken die Bewältigungsbemühungen einer Person oder gleichen bestimmte Defizite oder »blinde Flecken « im individuellen Verhalten durch die zusätzliche Unterstützung kompensatorisch aus.

Soziale Ressourcen wirken darüber hinaus ganz generell förderlich auf das Wohlbefinden, indem sie elementare, nicht situationsgebundene soziale Bedürfnisse erfüllen (Badura, 1981). Die Integration einer Person in ein System sozialer Beziehungen wirkt sich danach direkt förderlich auf ihr Wohlbefinden aus, da sie die grundsätzlichen Bedürfnisse nach Zugehörigkeit, Geborgenheit und sozialer Verortung befriedigt, die es ihr überhaupt erst ermöglichen, sich in ihrer Umwelt zurechtzufinden und ihrem Leben einen Sinn zu geben. Die unterstützende und sinnstiftende Bedeutung der sozialen Einbindung ist dem Individuum in ihrer weitreichenden Dimension oftmals gar nicht bewusst. Sie wird erst bei einem Verlust solcher sozialer Bezüge plötzlich spürbar, beispielsweise nach der Scheidung oder nach dem Ausstieg aus einem vorher in erster Linie als Belastung wahrgenommenen Berufsleben. Es handelt sich eher um scheinbar beiläufige Nebenprodukte und Begleiterscheinungen des alltäglichen Zusammenlebens.

Die besondere Stärke sozialer Ressourcen liegt generell in ihrer Vielfalt und ihrer Alltagsnähe. Sie reichen von alltäglichen und materiellen Hilfen, Geselligkeit, Rat, Information und Orientierung,

\section{Prof. Dr. Albert Lenz}

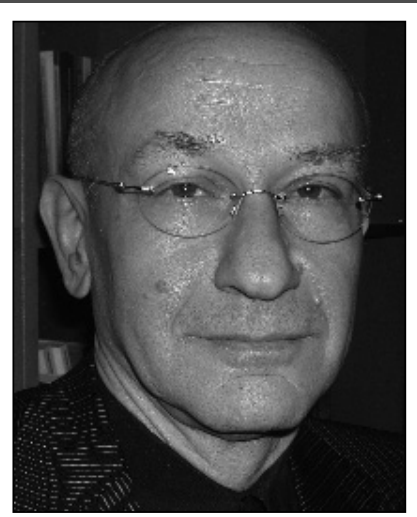

studierte Psychologie, Soziologie und Pädagogik an der Ludwig-Maximilians-Universität München und war dann leitender Psychologe an einer Erziehungsund Familienberatungsstelle. Seit dem Jahre 1994 ist er Hochschullehrer für Klinische Psychologie und Sozialpsychologie an der Katholischen Fachhochschule Nordrhein-Westfalen, Abteilung Paderborn, Fachbereich Sozialwesen. Er har eine Zusatzausbildung in Familientherapie und psychologischer Krisenintervention an der Akademie für Psychoanalyse und Psychotherapie München. Seine Arbeits- und Forschungsschwerpunkte sind Sozial- und Gemeindepsychiatrie, Psychiatrie und Jugendhilfe, Beratung und Krisenintervention, soziale Netzwerke und Empowerment, Theorie und Praxis der Gemeindepsychologie. Er ist Mitherausgeber der Zeitschrift »Praxis der Kinderpsychologie und Kinderpsychiatrie« (Verlag Vandenhoeck \& Ruprecht).

E-Mail a.lenz@kfhnw.de

bis hin zu Grundbedürfnissen wie die Vermittlung von sozialer Anerkennung, Geborgenheit, Zugehörigkeit, Liebe und Zuneigung. Klauer (2005) spricht von instrumenteller, kognitiver und emotionaler Unterstützung in spezifischen Lebenslagen und Problemsituationen, die Stabilität und Sicherheit vermitteln sowie Selbstwert und Selbstbewusstsein, also personale Ressourcen, sichern helfen.

Soziale Ressourcen sind zudem in die gemeinsamen alltäglichen sozialen Bezüge von Menschen eingebettet und ermöglichen daher eine schnelle Erreichbarkeit und Verfügbarkeit potenzieller Unterstützer. Man begegnet sich häufiger, trifft sich vielleicht sogar bei alltäglichen Anlässen. Dabei ergeben sich immer wieder Gelegenheiten zu Gesprächen, Fragen und sozialem Austausch, ohne sich sofort offen als hilfesuchende Person definieren zu müssen (vgl. Lenz, 2001).

\section{Soziale Netzwerke sind Möglichkeitsräume}

Soziale Netzwerke stellen zunächst ein latentes Potenzial dar, das es in Belastungssituationen durch geeignete Handlungen erst zu mobilisieren gilt. Mit dem Begriff der Mobilisierung sind alle von außen wahrnehmbaren Verhaltensaktivitäten einer belasteten Person gemeint, denen die Intention zu Grunde liegt, Unterstützungsleistungen aus dem sozialen Netzwerk zu evozieren (Klauer \& Winkeler, 2005). Das Mobilisierungsverhalten einer Person, das nicht nur direkte, sprachlich kodierte Appelle um Rat, Rückmeldung und Unterstützung, sondern auch indirekte Handlungsweisen wie mimischen Ausdruck, umfasst, wird offensichtlich durch allgemeine interpersonale Dispositionen und Stile des Hilfesuchens geprägt (Nadler, 1997). Tolsdorf (1976) spricht in diesem Zusammenhang von der subjektiven Netzwerkorientierung einer Person. Darunter versteht er ein Bündel von Überzeugungen, Einstellungen und Erwartungen, die sich auf die potenzielle Nützlichkeit der Netzwerkmitglieder beziehen, ihr bei der Bewältigung der Probleme und Krisen zu helfen.

Personen mit einer positiven Netzwerkorientierung beziehen sich viel häufiger auf Netzwerkmitglieder und mobilisieren in Krisensituationen soziale Unterstützung als Personen mit einer negativen 
Netzwerkorientierung. Tolsdorf (1976) fand in einer Studie heraus, dass die negative Netzwerkorientierung häufig geprägt war von Misstrauen und Vorbehalten gegenüber Netzwerkmitgliedern. Die betroffenen Personen empfanden es als unklug und gefährlich oder als Zeichen von Schwäche und Insuffizienz, ihr soziales Beziehungssystems bei der Krisenbewältigung in Anspruch zu nehmen. Es überwog die Einstellung, dass man mit seinen Problemen selber fertig werden müsse.

Die Mobilisierung sozialer Unterstützung setzt die Bereitschaft voraus, die Probleme oder Konflikte nach außen zu tragen, sie zu veröffentlichen (Lenz, 2001; 2005). Meist gehen diesem Schritt vielfältige Prozesse des Wahrnehmens, des Definierens und Bewertens voraus. Die Probleme werden in aller Regel zunächst normalisiert oder geleugnet, erst wenn diese Form der Kanalisierung der Empfindungen nicht mehr ausreicht, weil beispielsweise die Probleme immer sichtbarer wer- den, sie zunehmend häufiger auftreten oder die Person oder die Familie massiv beeinträchtigt ist, werden Problemdefinitionen und erste eigene Bewältigungsversuche unternommen. Erst wenn das eigene und innerfamiliäre Bewältigungshandeln gescheitert ist oder als nicht mehr ausreichend betrachtet wird, beginnen die Betroffenen allmählich ihre Probleme nach außen sichtbar zu machen und sich sinnvoll erkannte Bewältigungsstrategie einzusetzen. Die Gefühle der Ratlosigkeit, Hilflosigkeit und Anspannung sowie die damit einhergehenden körperlichen Begleitsymptomen wie Herzrasen, Atemnot, motorische Unruhe, Erschöpfung oder Schlafstörung führen darüber hinaus zu einer sich zunehmend verschlechternden psychischen und körperlichen Verfassung. Die Person zieht sich in der Folge

\section{"Soziale Ressourcen sind psychosoziale Schutzfaktoren"}

an Angehörige des sozialen Netzwerkes zu wenden.

In einer Krise läuft dieser Prozess der Hilfesuche nicht selten verzögert ab. Die Person ist durch den Verlust des seelischen Gleichgewichts und die akute Überforderung häufig unfähig, das Problem wahrzunehmen und eine bestimmte, als

\section{Netzwerkanalyse und die Netzwerkkonferenz}

Die Netzwerkforschung hat eine Vielzahl von Strategien und Interventionsformen entwickelt, die in der Krisenberatung von Bedeutung sind. Beispiele sind die Netzwerkanalyse und die Netzwerkkonferenz (vgl. dazu die ausführlichen Überblicke bei Lenz, 2000, 2005):

- Die verschiedenen Formen der Netzwerkrekonstruktion und Netzwerkanalyse stellen hilfreiche Medien zur Verdeutlichung und Bewusstmachung der sozialen Beziehungen im sozialen Umfeld dar. Die Person wird beispielsweise aufgefordert auf einer Netzwerkkarte, die aus konzentrischen Kreisen um ein in der Mitte gelegenes »Ich « besteht, Personen in Relation zu sich selbst einzutragen. Die visuelle Darstellung liefert Anstöße, um mit Erwachsenen, Kindern oder Jugendlichen über ihr Beziehungsnetz ins Gespräch zu kommen und damit eine vertiefte Analyse seiner Struktur, Qualität und Funktionen einzuleiten. Verluste und Veränderungen, wie Abhängigkeiten und kontrollierende Einmischungen, werden auf diese Weise sichtbarer. Wünsche nach mehr Nähe oder größerer Distanz, nach Unterstützung und Begleitung oder nach neuen Kontakten können klarer wahrgenommen werden. Durch die gezielte Auseinandersetzung tauchen verfügbare soziale Ressourcen auf, zugleich werden aber nicht selten auch diffuse Ängste, enttäuschte Hoffnungen, unerfüllt gebliebene Erwartungen und schmerzliche Erfahrungen mit Personen aus dem sozialen Netzwerk plötzlich wieder deutlich.

- Die Netzwerkkonferenz ist ein Instrument, um wichtige Teile eines sozialen Netzwerkes zu versammeln und gemeinsam eine Frage oder ein gemeinsames Problem zu bearbeiten. Das Ziel besteht darin, möglichst alle Personen, die mit dem Problem verknüpft sind, einzuladen und bislang wenig genutzte Kommunikationskanäle anzustoßen. Auf diese Weise sollen konstruktive Lösungen und Unterstützungspotenziale im sozialen Netzwerk aktiviert werden. Die Anwesenden sollen dabei ermutigt werden, Verantwortung für sich und ihre Angelegenheiten zu übernehmen sowie eigene Kräfte und Kompetenzen zu entdecken. Aufgabe des Krisenhelfers ist es dabei, diese netzwerkimmanenten Prozesse anzustoßen, zu katalysieren und für das Netzwerk nutzbar zu machen.

Albert Lenz

zurück und meidet soziale Situationen und Kontakte, was die Aktivierung und Nutzung sozialer Ressourcen erschwert, wenn nicht sogar verhindert.

Vieles spricht für die Annahme, dass die interpersonalen Dispositionen zur Veröffentlichung und im Hilfesuchverhalten wesentlich durch die individuelle Lebensgeschichte und vor allem durch die spezifischen Erfahrungen in der Herkunftsfamilie geformt wird. Durch individuell-biografische Erfahrungen in der Familie werden die Werte und Normen geprägt, was veröffentlicht werden kann, was nicht veröffentlich werden darf und wie man auf soziale Ressourcen zurückgreifen kann oder wie man es auf keinen Fall tun darf (Lenz, 2001).

Das Mobilisierungsverhalten zu stärken und die Person zu ermutigen, bei der Bewältigung der Probleme und Belastungen auf das Potenzial und Unterstützungsmöglichkeiten ihres sozialen Netzwerkes zurückzugreifen ist deshalb ein Handlungsprinzip professioneller Krisenberatung (vgl. beispielsweise Sonneck, 2000). Ziel der Förderung und Stärkung des Mobilisierungsverhaltens ist es, die Person anzuregen, Möglichkeiten und Potenziale im Netzwerk bewusster wahrzunehmen und individuelle Mobilisierungsstrategien $\mathrm{zu}$ entwickeln bzw. zu stärken (vgl. ausführlich Lenz, 2001; 2005).

\section{Aktivierung sozialer Ressourcen ist ein Handlungsprinzip von Krisenberatung}

Neben der gezielten Förderung des individuellen Mobilisierungsverhaltens 
sind eine Reihe von Methoden und Strategien zur unmittelbaren Netzwerkförderung und zur Initiierung neuer sozialer Netzwerke entwickelt worden (vgl. Röhrle et al., 1998; Lenz, 2001; 2005).

Mögliche Einsatzmöglichkeiten für diese Formen der Netzwerkförderung ergeben sich bei kleinen oder sehr reduzierten Beziehungsgefüge, in denen Segmente ganz auseinander gefallen sind oder wenn keine oder nur noch sehr rudimentäre soziale Beziehungen bestehen. Es kommt dabei darauf an, die Betroffenen zu einer bewussteren Auseinandersetzung auch mit den über die familiären Grenzen hin-

ausreichenden sozialen Beziehungen und deren Dynamik anzuregen, sie zu ermutigen, bestehende Bezüge zu intensivieren, neue Kontakte und Beziehungen aufzunehmen oder frühere Bindungen zu reaktivieren.

Die zentralen Ziele bestehen in der Suche und Analyse sozialer Beziehungen, in der Stärkung informeller Unterstützungsprozesse und damit in der Eröffnung und Erweiterung der Möglichkeitsspielräume einer Person.

\section{Literatur}

Badura, B. (1981). Zur sozialepidemiologischen Bedeutung sozialer Bindung und Unterstützung. In B. Badura (Hg.), Soziale Unterstützung und chronische Krankheit (S. 13-39). Frankfurt am Main: Suhrkamp.

Bergold, J. \& Schürmann, I. (2001). Krisenintervention - Neue Entwicklungen? Verhaltenstherapie und Psychosoziale Praxis, 1, 5-16.

Cullberg (1978). Krisen und Krisentherapie. Psychiatrische Praxis, 5, 25-34.

Diewald, M. (1991). Soziale Beziehungen: Verlust oder Liberalisierung? Soziale Unterstützung in informellen Netzwerken. Berlin: Sigma.

Keupp, H. (1987): Soziale Netzwerke Eine Metapher des gesellschaftlichen Umbruchs? In H. Keupp \& B. Röhrle (Hg.), Soziale Netzwerke (S. 11-53). Frankfurt am Main: Campus.

Klauer, T. (2005). Psychotherapie und soziale Unterstützung. Psychotherapeut, 6, 425-436.

Klauer, T. \& Winkeler, M. (2005). Mobilisierung sozialer Unterstützung: Konzepte, Befunde und Interventionsansätze. In U. Otto \& P. Bauer (Hg.), Mit Netzwerken professionell $\mathrm{zu}^{-}$ sammenarbeiten. Band 1: Soziale Netzwerke in Lebenslauf- und Lebenslagenperspektive (S. 157-180). Tübingen: dgvt.

Lenz, A. (2000). Praxis der netzwerkorientierten Trennungs- und Scheidungsberatung. In W. Körner \& G. Hörmann (Hg.), Handbuch der Erziehungsberatung. Band 2: Praxis der Erziehungsberatung (S. 91-124). Göttingen: Hogrefe.
Lenz, A. (2001). Partizipation von Kindern in Beratung und Therapie. Entwicklungen, Befunde und Handlungsperspektiven. Weinheim, München: Juventa.

Lenz, A. (2002). Empowerment und Ressourcenaktivierung. Perspektiven für die psychosoziale Praxis. In A. Lenz \& W. Stark (Hg.), Empowerment. Neue Perspektiven für psychosoziale Praxis und Organisation (S. 13-55). Tübingen: dgvt.

Lenz, A. (2005). Kinder psychisch kranker Eltern. Göttingen: Hogrefe.

Nadler, A. (1997). Personality and Help-seeking: Autonomous and dependent seeking of help. In G.R. Pierce, B. Lakey, I. G. Sarason \& B. R. Sarason (Eds.), Sourcebook of social support and personality (pp. 379-407). New York: Plenum Press.

Röhrle, B. (1994). Soziale Netzwerke und soziale Unterstützung. Weinheim, Psychologie Verlags Union.

Röhrle, B. Sommer, G. \& Nestmann; F. (Hg.) (1998). Netzwerkinterventionen. Tübingen dgvt.

Schwarzer, R./Leppin, A. (1989): Sozialer Rückhalt und Gesundheit. Göttingen.

Sonneck, G. (2000). Krisenintervention und Suizidverhütung. Wien: UTB Facultas.

Thoits, P. (1994): Stressors and problem-solving: The individual as psychological activist. Journal of Health and Social Behavior, 35, 143-159.

Tolsdorf, C.C. (1976). Social network, support, and coping: An exploration study. Family Process, 15, 407-417.

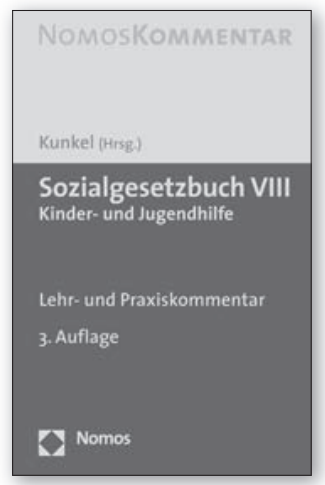

\section{Sozialgesetzbuch VIII}

Kinder- und Jugendhilfe Lehr- und Praxiskommentar Herausgegeben von Prof. PeterChristian Kunkel, FH Kehl 3. Auflage 2006, 1.119 S., geb., 79,- $€$, ISBN 978-3-8329-1380-9

Der Standard-Kommentar zum SGB VIII in Neuauflage: Das Gesetz zur Weiterentwicklung der Kinderund Jugendhilfe (KICK) ist selbstverständlich integriert.

\section{Der »Kunkel»}

- erläutert die Neuregelung der Inobhutnahme von Kindern und Jugendlichen,

- legt den Schutzauftrag des Jugendamtes dar,

- schafft Klarheit bei der Neuregelung der Leistungsvergabe für Kinder und Jugendliche sowie bei der Neuregelung der Kostenbeteiligung,

- zeigt umfassend den verstärkten Nachrang der Jugendhilfe im Verhältnis zu den Eltern auf.

Ebenfalls eingearbeitet ist das Tagesbetreuungsausbaugesetz (TAG) zur stärkeren Förderung von Kindertageseinrichtungen und der Kindertagespflege. Somit ist der Kommentar auch allen, die für den Ausbau des Förderangebots verantwortlich sind, ein wertvolles Hilfsmittel.

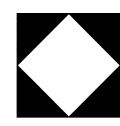

